\title{
ESPORTE PARALÍMPICO NA ESCOLA: REVISÃO BIBLIOGRÁFICA
}

PARALYMPIC SPORT AT SCHOOL: A LITERATURE REVIEW

DEPORTE PARALÍMPICO EN LA ESCUELA: REVISIÓN DE LITERATURA

Tiago Borgmann*, José Júlio Gavião de Almeida*

Palavras-chave

Revisão.

Educação especial. Educação Física.

Esportes.
Resumo: 0 esporte paralímpico é o principal meio de divulgação do esporte adaptado, presente também na escola, sendo o termo Educação Paralímpica agregador das atividades educacionais relacionadas a este movimento. As principais formas de inserção encontradas foram: o Dia Paralímpico Escolar (DPE), na Europa, e as aulas de Educação Física, no Brasil. Após levantamento bibliográfico de artigos originais, foram encontradas mudanças nos aspectos gerais relacionados à inclusão de alunos com deficiência em aulas de Educação Física. Esta temática ainda carece de estudos e propostas adequadas ao contexto cultural para fomentação do esporte paralímpico no âmbito acadêmico e escolar.

Abstract: Paralympic sport is the main means for disseminating disability sport, also present at school. The term Paralympic Education includes educational activities related to that movement. The main forms found were the Paralympic School Day (PSD) in Europe and Physical Education classes in Brazil. After a bibliographical review of original articles, changes were found in general aspects related to the inclusion of students with disabilities in Physical Education classes. The subject still lacks studies and proposals appropriate to the cultural context for fostering paralympic sport in the academic and school spheres.

Resumen: El deporte paralímpico es el principal medio de difusión de los deportes adaptados, también presentes en la escuela, siendo el término Educación Paralímpica el que engloba todas las actividades educativas relacionadas con este movimiento. Las principales formas de inserción encontradas fueron: el Día Paralímpico Escolar (DPE), en Europa, y las clases de Educación Física, en Brasil. Después de un levantamiento bibliográfico de artículos originales, fueron encontrados cambios en los aspectos generales relacionados con la inclusión de alumnos con discapacidad en clases de Educación Física. Este tema aún carece de estudios y propuestas adecuadas al contexto cultural para fomentar el deporte paralímpico en el ámbito académico y escolar
* Universidade Estadual de Campinas. Campinas, SP, Brasil.

E-mail: tiagoborgmann@ hotmail.com

Recebido em: 06-11-2013 Aprovado em: 17-11-2014 (c) (i) () Licence 


\section{INTRODUÇÃO}

O esporte paralímpico, principal meio de divulgação do esporte adaptado através das Paralimpíadas, maior evento em nível mundial voltado para pessoas com deficiência, está presente na sociedade contemporânea em diversos ambientes (MARQUES et al., 2009), assim como Special Olympics ${ }^{1}$, Deaflympics ${ }^{2}$ e modalidades esportivas adaptadas.

Devido ao sucesso dos Jogos Paralímpicos e o crescimento e desenvolvimento do Movimento Paralímpico, criou-se a necessidade de uma disciplina de ensino: Educação Paralímpica (INTERNATIONAL PARALYMPIC COMMITTEE, 2006), termo guarda-chuva para todas as atividades educacionais relacionadas ao movimento (KUDLÁČEK; JEŠINA; JANEČKA, 2009).

O Comitê Paralímpico Internacional incentiva atividades educacionais, científicas, culturais e pesquisas que contribuam para o desenvolvimento dos valores do Movimento Paralímpico, como o programa Paralympic School Day (PSD) - Dia Paralímpico Escolar (DPE) -, tendo como objetivo a criação de consciência e compreensão a respeito das pessoas com deficiência em crianças do ensino fundamental de todo o mundo (INTERNATIONAL PARALYMPIC COMMITTEE, 2006; PANAGIOTOU et al., 2008).

Esse programa foi implementado em alguns países europeus em 2003 (SCHELL, 2006), estruturado nos valores de respeito pela realização esportiva, respeito e aceitação das diferenças individuais, esporte como direito humano e capacitação e apoio social no esporte (INTERNATIONAL PARALYMPIC COMMITTEE, 2006; PANAGIOTOU; KUDLÁČEK; EVAGGELINOU, 2006; PANAGIOTOU et al., 2008).

Desta forma, este estudo objetivou encontrar as iniciativas nacionais e internacionais relacionadas à Educação Paralímpica, em artigos originais, manuais, resumos, teses e dissertações, escritos em língua inglesa e portuguesa, nos quais constavam a presença ou ensino do esporte paralímpico no ambiente escolar, através de uma pesquisa bibliográfica individualizada (GIL, 2002).

\section{EDUCAÇÃO PARALÍMPICA}

O termo Educação Paralímpica trouxe a premissa de integrar os ideais paralímpicos num sistema educacional formal, atuando como método pedagógico para desenvolver atitude positiva frente às pessoas com deficiência em crianças e jovens dentro das atividades das aulas de Educação Física escolar, enfatizando a importância do respeito pelas diferenças individuais (INTERNATIONAL PARALYMPIC COMMITTEE, 2006).

Assim, a Educação Paralímpica tem os seguintes objetivos: criar compreensão e desenvolver atitude positiva em relação às pessoas com deficiência; ajudar os jovens a compreender o direito ao desenvolvimento autônomo e igual participação; aumentar a conscientização sobre as ideias e valor educacional no Movimento Paralímpico; e apoiar e criar programas de educação e recursos em vários idiomas e formas de comunicação (INTERNATIONAL PARALYMPIC COMMITTEE, 2006).

1 Evento esportivo mundial voltado somente para pessoas com deficiência intelectual, realizado em período diferente ao dos Jogos Paralímpicos ou Olímpicos, com edições de verão e inverno. Possui objetivos, missão e visão próprios, e atletas competindo em divisões equitativas em cada modalidade, de acordo com o nível de habilidade individual, permitindo a todos a chance de ganhar (BRITTAIN, 2010).

2 Jogos mundiais para pessoas com deficiência auditiva, com edições de verão e inverno (DEPAUW; GAVRON, 2005). As modalidades disputadas são idênticas às competições convencionais, porém com os comandos sonoros substituídos por visuais (CRAFT; LIEBERMAN, 2004). 
O primeiro material relacionado a este termo foi The Paralympics: An overview of the Paralympic Games and people who participate, elaborado pelo Comitê Organizador dos Jogos de Atlanta 1996. O objetivo principal era criar uma consciência geral sobre pessoas com deficiência, destacando semelhanças, diferenças, incentivando estas pessoas a perceber 0 esporte como uma opção de lazer e de realização pessoal, e estimular o interesse pela atividade física como uma opção de vida para uma melhor saúde (INTERNATIONAL PARALYMPIC COMMITTEE, 2006).

Devido ao sucesso do primeiro material, para os jogos de Sydney 2000 foi preparado outro material, Set no Limits, com o principal objetivo de criar consciência e compreensão dos atletas com deficiência e gerar compromisso dos jovens australianos com os Jogos Paralímpicos, assim como para os jogos de inverno em Salt Lake City 2002 e Turim 2006, intitulados REACH e Kids Village, respectivamente. Para os jogos na cidade estado-unidense, o comitê organizador previu atividades e programas que proporcionassem oportunidades de aprendizagem para alunos e professores do estado de Utah. Já para a cidade italiana, o programa de Educação Paralímpica demonstrou grande sucesso antes e após a realização na promoção dos valores paralímpicos na região onde os Jogos foram realizados (INTERNATIONAL PARALYMPIC COMMITTEE, 2006).

Os Jogos Paralímpicos de Atenas 2004 marcaram a primeira colaboração ativa do Comitê Paralímpico Internacional na organização do programa The Paralympic Games from 1960 to 2004. O material tinha por objetivo ensinar a Educação Paralímpica para alunos do primário e secundário, educando-os para uma sociedade melhor (EVAGGELINOU, 2006; INTERNATIONAL PARALYMPIC COMMITTEE, 2006; SCHELL, 2006; XAFOPOULOS; KUDLÁČEK; EVAGGELINOU, 2009).

A Grécia foi o primeiro país a desenvolver não somente um material educacional para os Jogos, mas também introduzir no currículo escolar de todas as escolas, entre 2002 e 2003, uma hora extra de Educação Física chamada Educação Olímpica e Paralímpica. O programa tinha intenção de promover os valores olímpicos e paralímpicos e atitudes positivas em alunos sem deficiência e professores de Educação Física frente à inclusão de alunos com deficiência nas aulas (DOULKERIDOU; EVAGGELINOU; KUDLÁČEK, 2006; 2010; EVAGGELINOU, 2006).

Esse programa foi aplicado em 7.400 escolas do país, 1.000 no Chipre e 2.000 pelo mundo. Para a sua realização, mais de dois mil professores de Educação Física foram contratados para ensinar alunos do ensino fundamental e médio (EVAGGELINOU, 2006).

Dentro do programa educacional do Comitê Paralímpico do Reino Unido Ability vs. Ability foi lançado o material Get Set para os Jogos Olímpicos e Paralímpicos de Londres 2012 (MANSELL, 2010). Através de seus comitês paralímpicos nacionais, outros países lançaram seus programas de Educação Paralímpica, caso do Canadá, com Petro-Canada Paralympic School Programme, Austrália, com Telstra Paralympic Education Programme, e Bélgica, com School Project (INTERNATIONAL PARALYMPIC COMMITTEE, 2011a).

Com essas iniciativas mundiais, o principal meio de divulgação da Educação Paralímpica tem sido o Dia Paralímpico Escolar (DPE), uma das primeiras atividades educacionais não relacionadas aos jogos organizadas pelo Comitê Paralímpico Internacional (INTERNATIONAL PARALYMPIC COMMITTEE, 2006). 


\section{DIA PARALÍMPICO ESCOLAR}

Iniciado no final de 2003, a partir do interesse do Comitê de Ciência do Esporte do Comitê Paralímpico Internacional na introdução do Movimento Paralímpico nas escolas, o Dia Paralímpico Escolar (DPE) expandiu-se no ano seguinte após parceria com o Comitê Paralímpico Europeu e iniciação do projeto piloto de dois anos em seis países europeus (Alemanha, Bélgica, Grécia, Letônia, República Tcheca e Suécia) (SCHELL, 2006; PANAGIOTOU et al., 2008).

O DPE consiste em, através de um evento organizado dentro da escola, criar consciência e entendimento sobre as pessoas com deficiência e esporte, de maneira criativa, dinâmica, divertida e flexível. Esse conceito se desenvolveu e espalhou-se em diversas escolas pelo mundo (INTERNATIONAL PARALYMPIC COMMITTEE, 2006; XAFOPOULOS; KUDLÁČEK; EVAGGELINOU, 2009).

A partir da visão e a missão do Comitê Paralímpico Internacional, foram estabelecidos os seguintes valores para o Dia Paralímpico Escolar:

Quadro 1 - Valores estabelecidos para o Dia Paralímpico Escolar.

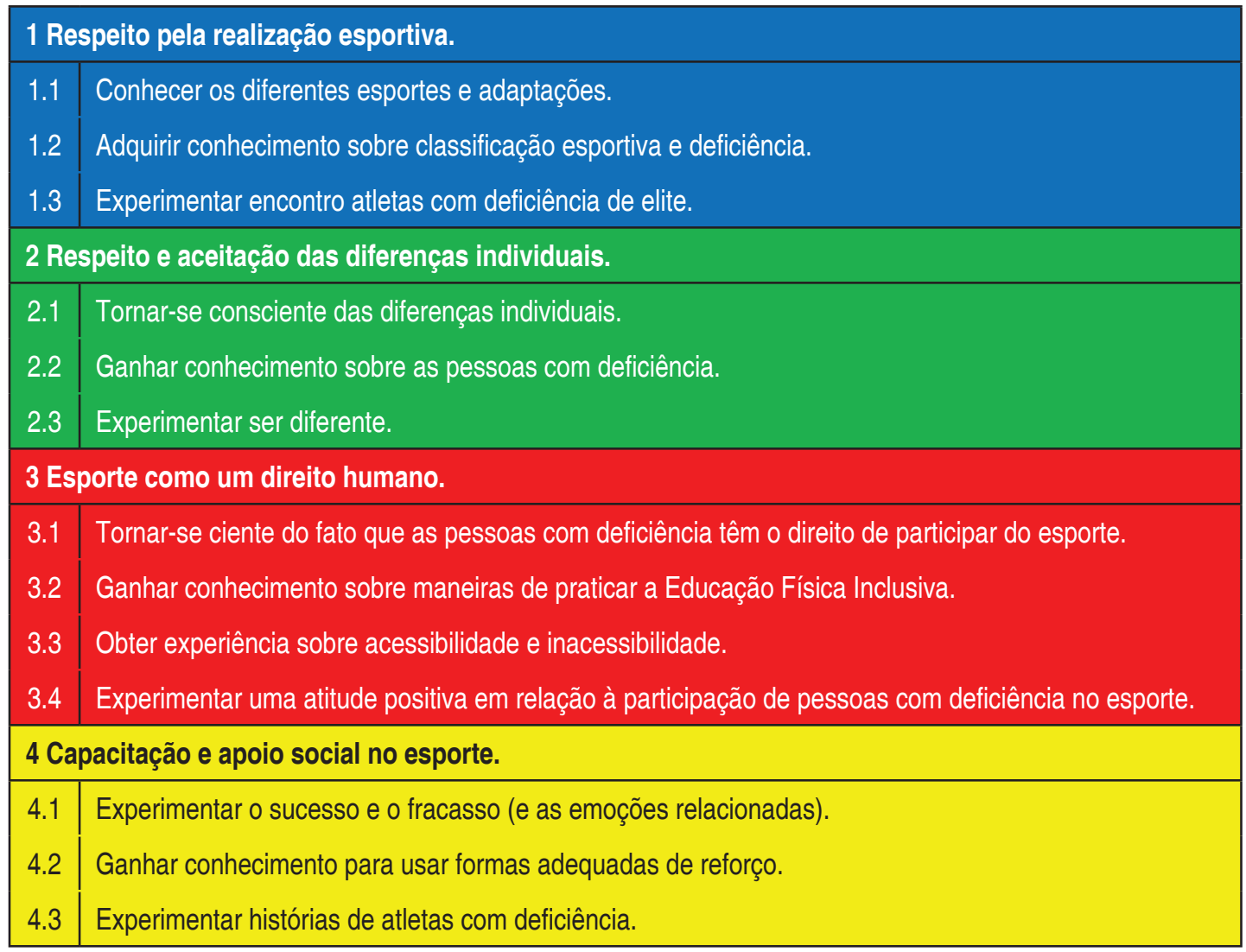

Fonte: INTERNATIONAL PARALYMPIC COMMITTEE. Paralympic School Day - Manual. 2006, p. 8. (traduzido).

Para cada um desses valores, foram estabelecidas algumas cores: azul - respeito pela realização esportiva; verde - respeito e aceitação das diferenças individuais; vermelho - esporte como um direito humano; e amarelo - capacitação e apoio social no esporte. Além disso, grupos de atividades são sugeridos para implementação do DPE, divididos entre os valores estabelecidos, tendo para cada uma das atividades um cartão explicativo enumerado (INTERNATIONAL PARALYMPIC COMMITTEE, 2006), conforme demonstrado no quadro abaixo: 
Quadro 2 - Atividades sugeridas para cada valor do Dia Escolar Paralímpico.

\begin{tabular}{|c|c|c|}
\hline N.ㅇ & Título e Atividade & Valores \\
\hline 01 & $\begin{array}{l}\text { Atletismo: } \\
\text { Praticando habilidades do Atletismo (campo e pista). }\end{array}$ & \multirow{7}{*}{$\begin{array}{l}\text { Respeito pela realização } \\
\text { esportiva. }\end{array}$} \\
\hline 02 & $\begin{array}{c}\text { Bocha: } \\
\text { Praticando habilidades da Bocha. }\end{array}$ & \\
\hline 03 & $\begin{array}{l}\text { Goalball: } \\
\text { Praticando habilidades do Goalball. }\end{array}$ & \\
\hline 04 & $\begin{array}{c}\text { Voleibol Sentado: } \\
\text { Praticando habilidades do Voleibol Sentado. }\end{array}$ & \\
\hline 05 & $\begin{array}{l}\text { Basquete em Cadeira de Rodas: } \\
\text { Praticando habilidades do Basquete em Cadeira de Rodas. }\end{array}$ & \\
\hline 06 & $\begin{array}{l}\text { Rúgbi em Cadeira de Rodas: } \\
\text { Praticando habilidades do Rúgbi em Cadeira de Rodas. }\end{array}$ & \\
\hline 07 & $\begin{array}{l}\text { Esportes de Inverno: } \\
\text { Ski Alpino, Ski Cross Country e Hóquei no Gelo. }\end{array}$ & \\
\hline 08 & $\begin{array}{l}\text { Um Conto de Fadas: } \\
\text { Discussão em classe sobre inclusão. }\end{array}$ & \multirow{5}{*}{$\begin{array}{l}\text { Respeito e aceitação das } \\
\text { diferenças individuais. }\end{array}$} \\
\hline 09 & $\begin{array}{l}\text { Visão: } \\
\text { Simulando cegueira ou deficiência visual. }\end{array}$ & \\
\hline 10 & $\begin{array}{l}\text { Jogo da Foto: } \\
\text { Experenciando similaridades e diferenças. }\end{array}$ & \\
\hline 11 & $\begin{array}{l}\text { Equipamento: } \\
\text { Pista de obstáculos - como usar o equipamento adaptado. }\end{array}$ & \\
\hline 12 & $\begin{array}{c}\text { Acessibilidade: } \\
\text { Descobrindo a acessibilidade da escola. }\end{array}$ & \\
\hline 13 & $\begin{array}{l}\text { Tênis de Mesa: } \\
\text { Praticando habilidades do Tênis de Mesa. }\end{array}$ & \multirow{5}{*}{$\begin{array}{l}\text { Esporte como um direito } \\
\text { humano. }\end{array}$} \\
\hline 14 & $\begin{array}{l}\text { Futebol: } \\
\text { Praticando habilidades do Futebol adaptado. }\end{array}$ & \\
\hline 15 & $\begin{array}{l}\text { Dança: } \\
\text { Praticando Dança inclusiva. }\end{array}$ & \\
\hline 16 & $\begin{array}{l}\text { Jogos Paralímpicos: } \\
\text { Vídeo de apresentação e discussão sobre os Jogos Paralímpicos. }\end{array}$ & \\
\hline 17 & $\begin{array}{l}\text { Quiz: } \\
\text { Adquirindo conhecimento sobre esportes e atletas paralímpicos. }\end{array}$ & \\
\hline 18 & $\begin{array}{l}\text { História de Atleta: } \\
\text { Encontro com atleta com deficiência. }\end{array}$ & \multirow{3}{*}{$\begin{array}{l}\text { Capacitação e apoio social no } \\
\text { esporte. }\end{array}$} \\
\hline 19 & $\begin{array}{l}\text { Classificação: } \\
\text { Examinando a classificação funcional no esporte paralímpico. }\end{array}$ & \\
\hline 20 & $\begin{array}{c}\text { Arte: } \\
\text { Refletindo através da arte. }\end{array}$ & \\
\hline
\end{tabular}

Fonte: INTERNATIONAL PARALYMPIC COMMITTEE. Paralympic School Day - Manual. 2006, p. 9. (traduzido). 
O evento divide-se em três fases: anterior, a realização e posterior ao DPE. A primeira fase tem duas missões: fornecer informações básicas aos participantes e organizar a implementação do evento na escola. A segunda fase é a concretização das atividades do Dia Paralímpico Escolar. Já na terceira fase, dá-se continuidade ao processo educacional, expandindo o conhecimento e a experiência adquirida nas atividades (INTERNATIONAL PARALYMPIC COMMITTEE, 2006).

No período que precede a realização do DPE, de no máximo dois meses, os professores de Educação Física devem preparar os alunos com informações corretas e realistas sobre as deficiências, ressaltando principalmente os direitos da população, sem enfoque médico. As atividades desenvolvidas devem ser de simulação e exemplos de como ser deficiente, valorizando as diferenças individuais, e em conjunto com o programa (INTERNATIONAL PARALYMPIC COMMITTEE, 2006).

Além desses aspectos, nesta fase faz-se necessário organizar a implementação do evento, abrangendo avaliar as condições de sua realização na escola (alunos, professores, "líderes", voluntários, ambiente e materiais), e planejar as atividades a serem desenvolvidas, como cerimonial de abertura e encerramento, seleção dos envolvidos e criação de grupos (INTERNATIONAL PARALYMPIC COMMITTEE, 2006).

Após os preparativos concluídos, realiza-se o Dia Paralímpico Escolar propriamente dito, levando-se em consideração na programação o tempo de transição entre os locais e a organização deles para os grupos subsequentes (INTERNATIONAL PARALYMPIC COMMITTEE, 2006), como mostra o exemplo no quadro a seguir.

Quadro 3 - Exemplo de programação para o Dia Paralímpico Escolar.

\begin{tabular}{|c|c|c|c|c|c|c|}
\hline GRUPOS & 1 & 2 & 3 & 4 & 5 & 6 \\
\hline & \multicolumn{6}{|c|}{ CERIMÔNIA DA ABERTURA } \\
\hline 45 minutos & \multicolumn{2}{|c|}{ Atletismo } & Bocha & Arte & Visão & Dança \\
\hline 45 minutos & Bocha & Visão & \multicolumn{2}{|c|}{ Atletismo } & Arte & Visão \\
\hline \multirow[t]{2}{*}{45 minutos } & Dança & Bocha & Visão & Dança & & \\
\hline & \multicolumn{6}{|c|}{ INTERVALO } \\
\hline 45 minutos & \multicolumn{2}{|c|}{ Quiz } & Arte & Visão & Dança & Bocha \\
\hline 45 minutos & Arte & Dança & & & Bocha & Arte \\
\hline \multirow[t]{2}{*}{45 minutos } & Visão & Arte & Dança & Bocha & & \\
\hline & \multicolumn{6}{|c|}{ CERIMÔNIA DE ENCERRAMENTO } \\
\hline
\end{tabular}

Fonte: INTERNATIONAL PARALYMPIC COMMITTEE. Paralympic School Day - Manual. 2006, p 14. (traduzido).

Assim como para os Jogos Paralímpicos existem os mascotes, símbolo de boa sorte, refletindo a cultura ou natureza do país anfitrião, o programa tem o Spirit the Bird, representando o "Espírito em Movimento", lema do Movimento Paralímpico, em referência ao espírito de superação dos próprios limites de cada atleta (INTERNATIONAL PARALYMPIC COMMITTEE, 2006).

Figura 1 - Mascote do Dia Paralímpico Escolar, Spirit the Bird.




Apesar do DPE ser realizado em um dia, é benéfica a continuidade da experiência, através de acompanhamento, ampliando a vivência e o conhecimento adquirido para situações reais. Deve-se levar em consideração a idade dos alunos, o ambiente e a cooperação dos professores, a fim de expandir o impacto do DPE, através de pesquisas e reflexões sobre 0 tema, levando em consideração os valores desenvolvidos (INTERNATIONAL PARALYMPIC COMMITTEE, 2006).

\section{ESPORTE PARALÍMPICO NA ESCOLA}

O esporte paralímpico foi desenvolvido em escolas de alguns países. Uma das primeiras iniciativas foi realizada em Atlanta, nos Estados Unidos, com duração de três anos, em virtude da realização dos Jogos Paralímpicos de 1996. Alunos com deficiência visual e física participaram das atividades juntamente com seus pares sem deficiência. As atividades, que consistiam em visualização de vídeos, participação em esportes paralímpicos e interação com mentores, eram regularmente programadas nas aulas de Educação Física, com duração de 45 a 55 minutos, com participação de todos os alunos da turma (WILHITE et al., 1997).

Através de outros estudos, percebeu-se a presença do esporte paralímpico nas escolas, principalmente no formato do DPE, de acordo com o manual proposto pelo Comitê Paralímpico Internacional (INTERNATIONAL PARALYMPIC COMMITTEE, 2006), em parceria com as universidades locais. Esses eventos foram organizados nas seguintes estruturas: dez estações de 15 minutos cada (PANAGIOTOU; KUDLÁČEK; EVAGGELINOU, 2006; PANAGIOTOU et al., 2008, 2009), seis estações de 40 minutos (VAN BIESEN; BUSCIGLIO; VANLANDEWIJCK, 2006; XAFOPOULOS; KUDLÁČEK; EVAGGELINOU, 2009), quatro estações principais com duas atividades cada (JEŠINA et al., 2006), três estações de 45 minutos cada (LIU; KUDLÁČEK; JEŠINA, 2010), e estações de 30 minutos (VIDAL et al., 2011).

Segundo o International Paralympic Committee (2006), durante a criação do kit do DPE e 35 implementações em seis países europeus diferentes, detectou-se que o tempo por atividade variou significativamente de 15 minutos a duas horas, porém, independentemente do tempo destinado para as atividades, deve-se considerar um tempo para a reflexão. Desta forma, sugere-se que a duração ideal de cada atividade seja de 40 a 45 minutos, como apresentado anteriormente no Quadro 3.

Atividades diferentes foram realizadas em cada estudo, porém estabelecidas pelo International Paralympic Committee (2006). Como exemplos, Panagiotou, Kudláček e Evaggelinou (2006) e Panagiotou et al. (2008) incluíram no programa dez atividades de intervenção: Direitos Humanos, Jogos Paralímpicos, Acessibilidade, Classificação, Voleibol Sentado, Goalball, Bocha Paralímpica, Basquete em Cadeira de Rodas, Pintura e Atletismo. Já Xafopoulos, Kudláček e Evaggelinou (2009) dividiram os participantes em seis grupos, os quais passavam pelas estações das seguintes atividades: Esportes Paralímpicos, Hóquei no Gelo, Mobilidade em Cadeira de Rodas, Basquete em Cadeira de Rodas, Encontro com atleta e Bocha.

Em outros estudos, o esporte paralímpico foi inserido na escola através de atividades práticas, dentro das aulas de Educação Física. No Brasil, em Campinas-SP, proporcionou-se a vivência de Goalball e Voleibol Sentado para crianças de 4르 série durante dois bimestres (SALERNO; ARAÚJO, 2008), e em Santa Maria-RS em 14 aulas foi oportunizada a vivência 
do Atletismo para deficientes visuais, Basquetebol em Cadeira de Rodas, Goalball e Voleibol Sentado para crianças da 3ํㅡㄹérie (LEHNHARD; PALMA, 2011).

Também no Brasil, Garcia (2009) desenvolveu atividades educativas de Goalball, Voleibol Sentado e Bocha Paralímpica com 61 alunos de duas turmas de 5aㅗ série (6ํano) de uma escola estadual de São Carlos-SP. Na mesma cidade, com enfoque pedagógico no desenvolvimento do Voleibol Sentado, Miron (2011) desenvolveu com 120 alunos de duas turmas de $5^{\text {a }}$ série e uma de $6^{\text {a }}$ série, de uma escola estadual, em oito encontros com cada turma, um programa de jogos e atividades lúdicas sobre a modalidade.

\section{ESTUDOS REALIZADOS E RESULTADOS APRESENTADOS}

Os estudos selecionados foram encontrados através de levantamento bibliográfico individual, sem a utilização de bases de dados, optando por artigos originais que possuíam relação com a inserção do esporte paralímpico na escola para crianças e jovens sem deficiência, redigidos em língua inglesa e portuguesa. Resumos de eventos, manuais e os trabalhos de conclusão de graduação, dissertações e teses foram descartados nesse momento, priorizando-se os artigos científicos, por apresentarem estudos na íntegra, assim como estrutura padrão, detalhes metodológicos aprofundados e avaliação em pares.

Quadro 4 - Estudos realizados sobre esporte paralímpico na escola (parte 1).

\begin{tabular}{|c|c|c|c|c|}
\hline $\begin{array}{l}\text { Autor(es)/ } \\
\text { Ano }\end{array}$ & Cidade/País & Objetivo(s) & Instrumento(s) & Sujeitos \\
\hline $\begin{array}{l}\text { Wilhite et } \\
\text { al., } 1997\end{array}$ & $\begin{array}{c}\text { Atlanta, Estados } \\
\text { Unidos. }\end{array}$ & $\begin{array}{l}\text { Avaliar o "Dia Paralím- } \\
\text { pico nas Escolas". }\end{array}$ & $\begin{array}{l}\text { Questionário } \\
\text { específico }\end{array}$ & $\begin{array}{l}704 \text { jovens (62 com e } 642 \\
\text { sem deficiência), com ida- } \\
\text { de entre } 11 \text { e } 21 \quad(14,2) \\
\text { anos. }\end{array}$ \\
\hline $\begin{array}{l}\text { Ješina et al., } \\
2006\end{array}$ & $\begin{array}{l}\text { Olomouc, Repú- } \\
\text { blica Tcheca. }\end{array}$ & $\begin{array}{l}\text { Avaliar o DPE na mu- } \\
\text { dança de atitudes fren- } \\
\text { te à inclusão de alunos } \\
\text { com deficiência. }\end{array}$ & $\begin{array}{l}\text { CAIPE-R e Ad- } \\
\text { jective Checklist }\end{array}$ & $\begin{array}{l}48 \text { crianças de } 4^{\underline{a}} \text { e } 5^{\text {a }} \text { sé- } \\
\text { ries, } 27 \text { meninos e } 21 \text { meni- } \\
\text { nas, com idade entre nove e } \\
12 \text {, e média de } 10,7 \text { e } 10,6 \\
\text { anos respectivamente. }\end{array}$ \\
\hline $\begin{array}{l}\text { Van Biesen; } \\
\text { Busciglio; } \\
\text { Vanlandewi- } \\
\text { jck, } 2006\end{array}$ & Bélgica & $\begin{array}{l}\text { Investigar a influência } \\
\text { do DPE, gênero, expo- } \\
\text { sição prévia e aspectos } \\
\text { competitivos nas atitu- } \\
\text { des de estudantes sem } \\
\text { deficiência frente à in- } \\
\text { clusão de alunos com } \\
\text { deficiência. }\end{array}$ & CAIPE-R & $\begin{array}{l}196 \text { estudantes, } 100 \text { meni- } \\
\text { nos e } 96 \text { meninas, de três } \\
\text { escolas primárias distintas, } \\
\text { com idades entre oito e } 13 \\
\text { anos. }\end{array}$ \\
\hline $\begin{array}{l}\text { Panagiotou; } \\
\text { Kudláček; } \\
\text { Evaggeli- } \\
\text { nou, } 2006\end{array}$ & Serres, Grécia. & $\begin{array}{l}\text { Analisar os efeitos do } \\
\text { DPE na mudança de } \\
\text { atitudes das crianças } \\
\text { frente à inclusão de } \\
\text { alunos com deficiência } \\
\text { nas aulas de Educação } \\
\text { Física. }\end{array}$ & CAIPE-R & $\begin{array}{l}247 \text { estudantes, } 117 \text { meni- } \\
\text { nos e } 130 \text { meninas, de } 5^{\text {a }} \\
\text { e } 6^{\text {a }} \text { séries de cinco esco- } \\
\text { las primárias urbanas, com } \\
\text { idade entre } 10 \text { a } 12 \text { anos, } \\
\text { sendo que } 178 \text { alunos ( } 86 \\
\text { do grupo experimental e } 92 \\
\text { do controle) eram de três } \\
\text { escolas não inclusivas e } 69 \\
\text { (32 do grupo experimental } \\
\text { e } 37 \text { do controle) eram de } \\
\text { duas escolas inclusivas. }\end{array}$ \\
\hline
\end{tabular}




Examinar os efeitos do
DPE nas atitudes e as
diferenças entre gêne-
ros de estudantes sem
Panagiotou Seficiência de $5^{\underline{a}}$ e $6^{\underline{a}}$
et al., 2008 Sérécia. $\quad$ destente à inclusão
de crianças com defi-
ciência nas aulas de
Educação Física.

Examinar os efeitos do DPE nas atitudes e as diferenças entre gênedeficiência de $5^{\underline{a}}$ e $6^{\underline{a}}$ séries frente à inclusão de crianças com defiEducação Física.
178 estudantes, 83 da $5^{\text {a }}$ série e 95 da 6ª série, sendo 86 meninos e 92 meninas, com média de idade geral de $11,53 \pm 0,5$, $11,48 \pm 0,5$ para os meninos e $11,59 \pm 0,5$ anos para as meninas, sendo que 86 eram do grupo experimental e 92 do grupo controle.

\begin{tabular}{|c|c|c|c|c|}
\hline $\begin{array}{c}\text { Salerno; } \\
\text { Araújo, } 2008\end{array}$ & Campinas, Brasil & $\begin{array}{l}\text { Propor o ensino de es- } \\
\text { portes adaptados nas } \\
\text { aulas de Educação Fí- } \\
\text { sica. }\end{array}$ & $\begin{array}{l}\text { Ficha de obser- } \\
\text { vação (diário de } \\
\text { classe) }\end{array}$ & $\begin{array}{l}\text { Alunos das turmas de } 4^{a} \\
\text { série. }\end{array}$ \\
\hline $\begin{array}{l}\text { Panagiotou } \\
\text { et al., } 2009\end{array}$ & $\begin{array}{l}\text { Serres e Florina, } \\
\text { Grécia. }\end{array}$ & $\begin{array}{l}\text { Analisar o impacto do } \\
\text { DPE nas atitudes de } \\
\text { alunos de } 5^{\underline{a}} \text { e } 6^{a} \text { séries } \\
\text { frente à inclusão de } \\
\text { alunos com deficiência } \\
\text { nas aulas de Educação } \\
\text { Física. }\end{array}$ & CAIPE-R & $\begin{array}{l}310 \text { alunos, } 148 \text { da } 5^{\text {a }} \text { e } \\
162 \text { da } 6^{\text {a }} \text { série, sendo } 143 \\
\text { meninos e } 167 \text { meninas, } \\
\text { de sete escolas primárias, } \\
\text { com média de idade entre } \\
11,52 \pm 0,5 \text { anos, sendo que } \\
181 \text { eram do grupo expe- } \\
\text { rimental e } 129 \text { do grupo } \\
\text { controle. }\end{array}$ \\
\hline $\begin{array}{l}\text { Xafopoulos; } \\
\text { Kudláček; } \\
\text { Evaggeli- } \\
\text { nou, } 2009\end{array}$ & $\begin{array}{l}\text { Ostrava, Repú- } \\
\text { blica Tcheca. }\end{array}$ & $\begin{array}{l}\text { Investigar os efeitos do } \\
\text { DPE nas atitudes de } \\
\text { crianças de uma escola } \\
\text { internacional frente à } \\
\text { inclusão de pares com } \\
\text { deficiência. }\end{array}$ & $\begin{array}{l}\text { CAIPE-R e Ad- } \\
\text { jective Checklist }\end{array}$ & $\begin{array}{l}71 \text { crianças, } 42 \text { meninos } \\
\text { e } 29 \text { meninas, com média } \\
\text { de idade de } 11,33 \pm 2,91 \text { e } \\
11,17 \pm 3,23 \text { anos respecti- } \\
\text { vamente. }\end{array}$ \\
\hline
\end{tabular}

\begin{tabular}{|c|c|c|c|c|}
\hline $\begin{array}{c}\text { Liu; Ku- } \\
\text { dláček; } \\
\text { Ješina, } 2010\end{array}$ & $\begin{array}{l}\text { Ostrava, Repú- } \\
\text { blica Tcheca. }\end{array}$ & $\begin{array}{l}\text { Determinar a influência } \\
\text { do DPE nas atitudes de } \\
\text { estudantes frente à in- } \\
\text { clusão de pessoas com } \\
\text { deficiência; } \\
\text { analisar as teorias da } \\
\text { Educação Paralímpica } \\
\text { em ambiente escolar. }\end{array}$ & $\begin{array}{l}\text { CAIPE-R e Ad- } \\
\text { jective Checklist }\end{array}$ & $\begin{array}{l}36 \text { crianças, } 17 \text { meninos } \\
\text { e } 19 \text { meninas, com média } \\
\text { de idade de } 11,88 \pm 0,49 \text { e } \\
11,68 \pm 0,48 \text { anos, respecti- } \\
\text { vamente. }\end{array}$ \\
\hline
\end{tabular}

Fonte: quadro elaborado pelos autores.

No Quadro 4, encontram-se os estudos realizados sobre a presença do esporte paralímpico na escola, assim como as cidades e países onde foram concretizados, objetivos propostos, instrumentos utilizados e descrição dos sujeitos de cada pesquisa. Já no quadro seguinte, encontram-se a metodologia utilizada e os principais resultados encontrados nesses mesmos estudos. 
Quadro 5 - Estudos realizados sobre esporte paralímpico na escola (parte 2).

\begin{tabular}{ccl}
\hline Autor(es)/Ano & \multicolumn{1}{c}{ Metodologia } & \multicolumn{1}{c}{ Resultados } \\
\hline $\begin{array}{c}\text { Wilhite et al., } \\
1997\end{array}$ & $\begin{array}{c}\text { Pré e pós-teste, com intervalo de uma } \\
\text { semana da intervenção. }\end{array}$ & $\begin{array}{l}\text { Compromisso com esporte e lazer de todos; } \\
\text { alunos com deficiência foram menos propen- } \\
\text { sos a concordar com a importância da sua } \\
\text { participação nas atividades com seus cole- } \\
\text { gas sem deficiência. }\end{array}$ \\
\hline $\begin{array}{c}\text { Ješina et al., } \\
\text { 2006 }\end{array}$ & Pré-teste, intervenção e pós-teste. & $\begin{array}{l}\text { Efeitos positivos do DPE, sendo médio no } \\
\text { CAlPE-CZ e pequeno no Adjective Checklist. }\end{array}$ \\
\hline $\begin{array}{c}\text { Van Biesen; Bus- } \\
\text { ciglio; Vanlande- } \\
\text { wijck, 2006 }\end{array}$ & Pré e pós-teste, com intervalo de uma \\
semana da intervenção. & $\begin{array}{l}\text { Duas escolas obtiveram efeitos positivos; } \\
\text { meninas apresentaram atitudes mais positi- } \\
\text { vas que meninos; } \\
\text { alunos mais competitivos apresentaram me- } \\
\text { nor pontuação; } \\
\text { experiência prévia com pessoas com defi- } \\
\text { ciência não apresentou influência nas atitu- } \\
\text { des positivas frente à inclusão. }\end{array}$ \\
& &
\end{tabular}

Panagiotou; Ku-
dláček; Evaggelidláček; Evaggeli- Pré-teste, intervenção e pós-teste. nou, 2006

\begin{tabular}{|c|c|}
\hline $\begin{array}{l}\text { Panagiotou et } \\
\text { al., } 2008\end{array}$ & $\begin{array}{l}\text { Pré e pós-teste, com duas semanas de } \\
\text { intervalo. }\end{array}$ \\
\hline
\end{tabular}

Escolas não inclusivas: efeito positivo nas atitudes de crianças sem deficiência nos aspectos gerais; escolas inclusivas: diminuição não significativa no escore das atitudes gerais dos alunos.

Diferenças positivas no grupo experimental nas atitudes gerais após o DPE; sem efeitos diferentes entre meninos e meninas.

Proposta de ensino dos esportes adaptados na escola.

Diferença estatística no grupo experimental somente nas atitudes gerais, enquanto não houve diferenças nas atitudes específicas.

\section{Xafopoulos; Ku- dláček; Evaggeli- nou, 2009}

Panagiotou et al., 2009

Pré-teste, intervenção e pós-teste.
Diferenças significativas somente nas atitudes gerais entre meninas, através do Adjective Checklist; outras mudanças não foram significativas.
Liu; Kudláček; Ješina, 2010
Pré e pós-teste, com intervalo de uma semana da intervenção.
Meninas com atitude mais positiva frente às pessoas com deficiência; diminuição do escore das atitudes frente às adaptações de regras esportivas.

Fonte: quadro elaborado pelos autores.

\section{DISCUSSÃO}

Procurando atingir os objetivos propostos ao estudo, encontraram-se iniciativas relacionadas à Educação Paralímpica e à presença do esporte paralímpico nas aulas de Educação Física em artigos científicos, materiais didáticos, anais de eventos, trabalhos de conclusão de curso, dissertações e teses. 
O Dia Paralímpico Escolar (DPE), principal forma de divulgação da Educação Paralímpica, necessita de atualizações e revisões no programa em virtude de estudos realizados, já que as primeiras iniciativas foram em 2003 e o manual (INTERNATIONAL PARALYMPIC COMMITTEE, 2006) lançado em 2006. Da mesma forma, além de estar presente na escola no formato de evento (WILHITE et al., 1997; JEŠINA et al., 2006; VAN BIESEN; BUSCIGLIO; VANLANDEWIJCK, 2006; PANAGIOTOU; KUDLÁČEK; EVAGGELINOU, 2006; PANAGIOTOU et al., 2008, 2009; XAFOPOULOS; KUDLÁČEK; EVAGGELINOU, 2009; LIU; KUDLÁČEK; JEŠINA, 2010; VIDAL et al., 2011), pode-se desenvolver a perspectiva de utilização do esporte paralímpico como conteúdo do programa curricular da Educação Física, como se tem visto em algumas iniciativas brasileiras (SALERNO; ARAÚJO, 2008; GARCIA, 2009; LEHNHARD; PALMA, 2011; MIRON, 2011).

Dentre os artigos encontrados, percebe-se a existência de grande maioria de origem europeia, com predominância dos países que iniciaram com o programa DPE - Grécia (PANAGIOTOU; KUDLÁČEK; EVAGGELINOU, 2006; PANAGIOTOU et al., 2008, 2009), Bélgica (VAN BIESEN; BUSCIGLIO; VANLANDEWIJCK, 2006) e República Tcheca (JEŠINA et al., 2006; XAFOPOULOS; KUDLÁČEK; EVAGGELINOU, 2009; LIU; KUDLÁČEK; JEŠINA, 2010). De forma geral, esses estudos tinham por objetivo avaliar os efeitos do evento nas mudanças de atitudes frente à inclusão de alunos com deficiência nas aulas de Educação Física.

Nos demais países, destaca-se os Estados Unidos (WILHITE et al., 1997), como pioneiro na promoção do esporte adaptado na escola para pessoas com e sem deficiência e com objetivo semelhante aos estudos europeus, e o Brasil (SALERNO, ARAÚJO, 2008), com propostas de promoção do ensino das modalidades paralímpicas também para crianças sem deficiência na escola.

Nos estudos voltados principalmente para os efeitos do programa DPE em crianças sem deficiência frente à inclusão de alunos com deficiência em aulas de Educação Física foram utilizados para avaliação o Children's Attitudes toward Integrated Physical Education - Revised (CAIPE-R) (BLOCK, 1995) e o Siperstein's Adjective Checklist (SIPERSTEIN, 1980 apud JEŠINA et al., 2006).

O Children's Attitudes toward Integrated Physical Education - Revised (CAIPE-R) é um questionário validado nos Estados Unidos utilizado para avaliar as atitudes de crianças sem deficiências frente à inclusão de colegas com deficiências nas aulas de Educação Física Escolar, nos aspectos gerais e esportivo-específicos (BLOCK, 1995). Possui versões adaptadas às realidades esportivas e culturais europeias (basquetebol em substituição ao beisebol nas questões esportivo-específicas de 9 a 13 da versão original), em consulta ao autor do CAIPE-R e aprovada por três especialistas da área na Europa (PANAGIOTOU; KUDLÁČEK; EVAGGELINOU, 2006), aplicado na República Tcheca (CAIPE-CZ) (JEŠINA et al., 2006; XAFOPOULOS; KUDLÁČEK; EVAGGELINOU, 2009; LIU; KUDLÁČEK; JEŠINA, 2010), Grécia (PANAGIOTOU; KUDLÁČEK; EVAGGELINOU, 2006; PANAGIOTOU et al., 2008, 2009), Portugal (CAMPOS; NOBRE; FERREIRA, 2010) e Bélgica (VAN BIESEN; BUSCIGLIO; VANLANDEWIJCK, 2006).

Já o Siperstein's Adjective Checklist, desenvolvido para avaliar os julgamentos dos atributos de crianças para com colegas com deficiência, baseado entre a escolha de 34 adjetivos, revela opiniões e sentimentos, positivos e negativos (JEŠINA et al., 2006). Esse instrumento foi 
aplicado na República Tcheca (JEŠINA et al., 2006; XAFOPOULOS; KUDLÁČEK; EVAGGELINOU, 2009; LIU; KUDLÁČEK; JEŠINA, 2010).

Alguns instrumentos de pesquisa foram criados especificamente para atingir os objetivos do estudo sobre esporte paralímpico em questão, como o questionário (WILHITE et al., 1997) e a ficha de observação (SALERNO; ARAÚJO, 2008).

De forma geral, os sujeitos das pesquisas foram meninos e meninas europeus sem deficiência da $4^{\underline{a}}$ a $6^{\underline{a}}$ séries, com idade entre oito e 13 anos. A única exceção foi 0 estudo de Wilhite et al. (1997), o qual apresentou idades até 21 anos e crianças norte-americanas com e sem deficiência.

Em relação à metodologia utilizada nos artigos, percebeu-se predominância dos autores na utilização da pesquisa experimental (THOMAS; NELSON, 2002) de pré e pós-testes, com realização do DPE, porém em diferentes formatos. Os estudos de Panagiotou, Kudláček e Evaggelinou (2006) e Panagiotou et al. (2008; 2009) apresentaram estruturas semelhantes, com aplicação do CAIPE-R antes e após a intervenção nos grupos experimentais e aulas de Educação Física nos grupos controle, utilizando a mesma estrutura de DPE nos três estudos.

Sendo o único estudo que abrangeu o esporte adaptado como conteúdo das aulas Educação Física, Salerno e Araújo (2008) apresentaram uma proposta de ensino aplicável ao contexto escolar, com participação dos alunos, pesquisa sobre o tema e formulação de conceitos, além de vivência das modalidades Goalball e Voleibol Sentado.

Os resultados do DPE mostraram efeitos positivos após a implementação do programa em todos os casos, porém essas mudanças foram nos aspectos gerais relacionados à inclusão de alunos com deficiência nas aulas de Educação Física (JEŠINA et al., 2006; VAN BIESEN; BUSCIGLIO; VANLANDEWIJCK, 2006; PANAGIOTOU; KUDLÁČEK; EVAGGELINOU, 2006; PANAGIOTOU et al., 2008; 2009; XAFOPOULOS; KUDLÁČEK; EVAGGELINOU, 2009). Segundo Loovis e Loovis (1997), programas que incluem informações, jogos e discussão em grupo sobre deficiência podem contribuir para a mudança de atitudes de crianças sem deficiência.

Houve alterações significativamente negativas nos aspectos esportivos específicos, relacionados a adaptações de regras (LIU; KUDLÁČEK; JEŠINA, 2010), pois, segundo Kalyvas e Reid (2003), as crianças não aceitaram adaptações de regras nos esportes por ser menos desafiador e interessante.

Também foram encontrados efeitos positivos maiores nas atitudes das meninas do que nas dos meninos através do CAIPE-R (VAN BIESEN; BUSCIGLIO; VANLANDEWIJCK, 2006; LIU; KUDLÁČEK; JEŠINA, 2010) e do Adjective Checklist (XAFOPOULOS; KUDLÁČEK; EVAGGELINOU, 2009), assim como apresentado em estudos anteriores (TRIPP; FRENCH; SHERRILL, 1995; LOOVIS; LOOVIS, 1997; SLININGER; SHERRILL; JANKOWSKI, 2000). Porém, Panagiotou et al. (2008) não encontraram diferenças entre meninos e meninas frente à inclusão de alunos com deficiência nas aulas de Educação Física, assim como em Kalyvas e Reid (2003).

Através dos resultados dos artigos analisados, alunos mais competitivos obtiveram menor escore no CAIPE-R (VAN BIESEN; BUSCIGLIO; VANLANDEWIJCK, 2006), por acreditar que a atividade diminuiu de intensidade com um participante com menor habilidade (BLOCK, 
$2000^{3}$ apud VAN BIESEN; BUSCIGLIO; VANLANDEWIJCK, 2006). Também não houve influência da exposição prévia a pessoas com deficiência nas atitudes frente à inclusão de alunos com deficiência nas aulas de Educação Física (VAN BIESEN; BUSCIGLIO; VANLANDEWIJCK, 2006), em desacordo com a hipótese do mesmo estudo.

Com a utilização dos esportes paralímpicos na escola, envolvendo política, cultura e prática inclusiva de longo prazo, como proposto por Salerno e Araújo (2008), surge uma alternativa para melhorar as atitudes das crianças frente à inclusão de alunos com deficiência nas aulas de Educação Física e, consequentemente, aceitação das adaptações de regras nas atividades práticas, principalmente por parte dos meninos, como encontrado em alguns estudos (JEŠINA et al., 2006; VAN BIESEN; BUSCIGLIO; VANLANDEWIJCK, 2006; PANAGIOTOU; KUDLÁČEK; EVAGGELINOU, 2006; PANAGIOTOU et al., 2008; 2009; XAFOPOULOS; KUDLÁČEK; EVAGGELINOU, 2009; LIU; KUDLÁČEK; JEŠINA, 2010).

\section{CONCLUSÃO}

O esporte paralímpico desenvolve-se gradativamente no cenário mundial, nos contextos competitivo, tecnológico, acadêmico e educacional. Este último, foco do presente estudo, é percebido como âmbito recente e com grande potencial de exploração e expansão.

Em virtude da Educação Paralímpica ser um termo timidamente conhecido, ainda carece de divulgação internacional e nacional, de forma que possa alcançar todos os âmbitos relacionados ao esporte paralímpico, principalmente o acadêmico, para que novos estudos sejam realizados, e o termo massificado.

A presença do esporte paralímpico nas escolas busca sua identidade, assim como a Educação Paralímpica, adequada aos contextos cultural, escolar e nacional. Os resultados dos estudos já realizados apontaram efeitos positivos nos aspectos gerais da inserção do esporte paralímpico na escoa frente à inclusão de alunos com deficiência através do DPE. A possibilidade de utilização de outros mecanismos que possam gerar mudanças significativas nos aspectos esportivos, referentes às adaptações de regras, assim como a criação de cultura, política e práticas inclusivas na escola, ainda não foram avaliados, mesmo sabendo-se de experiências bem-sucedidas.

A inserção do esporte paralímpico na escola carece de estudos nesse ambiente, através de propostas diferenciadas, seja como evento ou ensino nas aulas de Educação Física, com modalidades esportivas, estruturado pedagogicamente para contribuir na formação dos alunos em todos os aspectos, fomentando o esporte paralímpico no âmbito acadêmico e escolar.

\section{REFERÊNCIAS}

BLOCK, Martin E. Development and Validation of Children's Attitudes toward Integrated Physical Education-Revised (CAIPE-R) Inventory. Adapted Physical Activity Quarterly, Champaign, v.12, p. 60-77, 1995.

BRITTAIN, lan. The Paralympic Games Explained. London: Routledge, 2010.

CAMPOS, Maria João; NOBRE, Moacyr; FERREIRA, José Pedro. Attitudes of 14 to 16 years old students without disabilities towards inclusion in physical education. In: EUROPEAN CONGRESS OF

3 BLOCK, Martin E. Including children with disabilities in regular physical education: a guide for practitioners. $2^{\text {nd }}$ ed.. Baltimore: Brooks 2000. p.104- 231. 
ADAPTED PHYSICAL ACTIVITY, 2010, Jyväskylä, Finland. Book of Abstract... Jyväskylä: EUFAPA, 2010. p. 34.

CRAFT, Diane H.; LIEBERMAN, Lauren. Deficiência Visual e Surdez. In: WINNICK, J. P. Educação física e esportes adaptados. Tradução [da 3. ed. original] de Fernando Augusto Lopes. Barueri: Manole, p. 181-206, 2004.

DEPAUW, Karen P.; GAVRON, Susan J. Disability Sport. $2^{\text {nd }}$ ed.. Champaign: Human Kinetics, 2005. DOULKERIDOU, Agapi; EVAGGELINOU, Christina; KUDLÁČEK, Martin. Attitudes of Greek physical education teachers towards inclusion of students with disabilities in physical education classes. In: EUROPEAN CONGRESS OF ADAPTED PHYSICAL ACTIVITY, 8. 2006, Olomouc, Czech Republic. Proceedings... Olomouc: 2006.

DOULKERIDOU, Agapi; EVAGGELINOU, Christina; KUDLÁČEK, Martin. Components of attitudes towards the inclusion of students with disabilities in physical education in the ATIPDPE-GR instrument for Greek physical educators. Acta Universitatis Palackianae Olomucensis, Gymnica, Olomouc, v. 40, n. 4, p. 63-68, 2010.

EVAGGELINOU, Christina. Creating a school for all in Greece: the model of Paralympic education. In: EUROPEAN CONGRESS OF ADAPTED PHYSICAL ACTIVITY, 8. 2006, Olomouc, Czech Republic. Proceedings... Olomouc: EUFAPA, 2006.

GARCIA, Flávia Rossi. Esportes adaptados e educação física escolar: implicações para uma prática pedagógica inclusiva. 2009, 56f. Monografia de Conclusão de Curso (Graduação em Educação Física) Centro de Educação e Ciências Humanas, Universidade Federal de São Carlos, São Carlos, 2009.

GIL, Antônio Carlos. Como elaborar projetos de pesquisa. 4. ed. São Paulo: Atlas, 2002.

INTERNATIONAL PARALYMPIC COMMITTEE. Paralympic Games Education Programmes.

Disponível em: < http://www.paralympic.org/ThelPC/WWD/ParalympicGamesEducationProgrammes >. Acesso em: 15 ago. 2011a.

INTERNATIONAL PARALYMPIC COMMITTEE. Paralympic School Day. Disponível em: < http:// www.paralympic.org/ThelPC/WWD/ParalympicSchoolDay >. Acesso em: 15 ago. 2011b.

INTERNATIONAL PARALYMPIC COMMITTEE. Paralympic School Day: Manual. Bonn, 2006. p. 46.

JEŠINA, Ondrej et al. Effect of an intervention program on attitude of elementary school children toward inclusion of children with disability. In: EUROPEAN CONGRESS OF ADAPTED PHYSICAL ACTIVITY, 8. 2006, Olomouc, Czech Republic. Proceedings... Olomouc: EUFAPA, 2006.

KALYVAS, Vassilis, REID, Greg. Sport Adaptation, Participation, and Enjoyment of Students With and Without Physical Disabilities. Adapted Physical Activity Quarterly, Champaign, v. 20, p.182-199, 2003.

KUDLÁČEK, Martin; JEŠINA, Ondřej; JANEČKA, Zbyněk. Paralympijské Vzdělávací Programy. Tělesná Kultura, Olomouc, v.32, n. 1, p. 44-55, 2009.

LEHNHARD, Aline Rosso; PALMA, Luciana Erina. A organização do esporte adaptado nas aulas de educação física de séries iniciais do ensino fundamental: um relato de experiência. [Resumo] In: CONGRESSO PARAOLÍMPICO BRASILEIRO, 2 E CONGRESSO PARADESPORTIVO INTERNACIONAL, 1. 2011, Uberlândia. Anais... Uberlândia: Ministério do Esporte, 2011. p. 212-213.

LIU, Yang; KUDLÁČEK, Martin; JEŠINA, Ondřej. The influence of Paralympic School Day on children's attitudes towards people with disabilities. Acta Universitatis Palackianae Olomucensis, Gymnica, Olomouc, v. 40, n. 2, p. 63-69, 2010.

LOOVIS, E. Michael; LOOVIS, Cynthia Loeb. A disability awareness unit in physical education and attitudes of elementary school disabilities. Perceptual and Motor Skills, Missoula, v.84, p. 3, p.768770, 1997. 
MANSELL, Martin. Paralympics and inclusion on whose terms working towards a more equitable society. In: EUROPEAN CONGRESS OF ADAPTED PHYSICAL ACTIVITY, 2010, Jyväskylä, Finland. Book of Abstract... Jyväskylä: EUCAPA, 2010. p. 84.

MARQUES, Renato Francisco Rodrigues et al. Esporte olímpico e paraolímpico: coincidências, divergências e especificidades numa perspectiva contemporânea. Revista Brasileira de Educação Física e Esporte, São Paulo, v. 23, n. 24, p. 365-77, out./dez. 2009.

MIRON, Edison Martins. Da pedagogia do jogo ao voleibol sentado: possibilidades inclusivas na Educação Física Escolar. 2011, 340f. Tese (Doutorado em Educação Especial) - Centro de Educação e Ciências Humanas, Universidade Federal de São Carlos, São Carlos, 2011.

PANAGIOTOU, Anna et al. Evaluation of Student's Attitudes from Conventional Primary Schools toward Inclusion of Children with Disabilities in Physical Education after Implementation of a Program. Inquiries in Sport \& Physical Education, Thessaly, v.7, n.2, p. 103-113, 2009.

PANAGIOTOU, Anna K. et al. Attitudes of $5^{\text {th }}$ and $6^{\text {th }}$ grade Greek students toward the inclusion of children with disabilities in physical education classes after a Paralympic education program. European Journal of Adapted Physical Activity, Olomouc, v.1, n.2, p. 31-43, 2008.

PANAGIOTOU, Anna K.; KUDLÁČEK, Martin.; EVAGGELINOU, Christina. The effect of the implementation of the "Paralympic School - Day" program on the attitudes of primary school children towards the inclusion of children with disabilities in physical education. Studia Kinanthropologica, České Budějovice, v. 7, n. 2, p. 83-87, 2006.

SALERNO, Marina Brasiliano; ARAÚJO, Paulo Ferreira de. Esporte Adaptado como tema da Educação Física Escolar. Conexões, Campinas, v.6, ed. especial, p. 212-221, 2008.

SCHELL, Bart. IPC's Paralympic Education: projects and approaches. In: EUROPEAN CONGRESS OF ADAPTED PHYSICAL ACTIVITY, 8 .2006, Olomouc, Czech Republic. Proceedings... Olomouc; EUFAPA, 2006.

SLININGER, David; SHERRILL, Claudine; JANKOWSKI, Catherine M. Children's attitudes toward peers with severe disabilities: revisiting contact theory. Adapted Physical Activity Quarterly, Champaign, v.17, n.2, p.176-196, 2000.

THOMAS, Jerry R.; NELSON, Jack. K. Métodos de Pesquisa em Atividade Física. Porto Alegre: Artmed, 2002.

TRIPP, April; FRENCH, Ron; SHERRILL, Claudine. Contact theory and attitudes of children in Physical Education programs toward peers with disabilities. Adapted Physical Activity Quarterly, Champaign, v.12, n.4, p.323-332, 1995.

VAN BIESEN, D.; BUSCIGLIO, A.; VANLANDEWIJCK, Y. Attitudes towards inclusion of children with disabilities: the effect of implementation of "A Paralympic School Day" on Flemish elementary children. In: EUROPEAN CONGRESS OF ADAPTED PHYSICAL ACTIVITY, 8. 2006, Olomouc, Czech Republic. Proceedings... Olomouc: EUFAPA, 2006.

VIDAL, Maria Helena Candelori et al. Dia Escolar Paraolímpico: uma proposta de divulgação do mundo paraolímpico. [Resumo] In: CONGRESSO PARAOLÍMPICO BRASILEIRO, 2 E CONGRESSO PARADESPORTIVO INTERNACIONAL, 1. 2011. Uberlândia/MG, Brasil. Anais... Uberlândia: Ministério do Esporte, 2011. p. 78-79.

WILHITE, Barbara et al. Promoting Inclusive Sport and Leisure Participation: Evaluation of the Paralympic Day in the Schools Model. Adapted Physical Activity Quarterly, Champaign, v.14, p.131-146, 1997.

XAFOPOULOS, Georgios; KUDLÁČEK, Martin; EVAGGELINOU, Christina. Effect of intervention program "Paralympic School Day" on attitudes of children attending international school towards inclusion of students with disabilities. Acta Universitatis Palackianae Olomucensis, Gymnica, Olomouc, v. 39, n. 4, p. 63-71, 2009. 
Apoio: Conselho Nacional de Desenvolvimento Científico e Tecnológico (CNPq).

(J) Movimento, Porto Alegre, v. 21, n. 1, p. 53-68, jan./mar. de 2015. 